\title{
A EXPANSÃO DA COVID-19 NA BAIXADA FLUMINENSE - RJ: SEUS CAMINHOS E EFEITOS SOCIAIS NA PERIFERIA
}

\author{
THE EXPANSION OF COVID-19 IN BAIXADA FLUMINENSE - RJ: THEIR PATHS AND SOCIAL EFFECTS ON THE \\ PERIPHERY
}

\author{
LA EXPANSIÓN DE LA COVID-19 EN LA BAIXADA FLUMINENSE - RJ: SUS CAMINOS Y EFECTOS SOCIALES EN LA \\ PERIFERIA
}

\section{RESUMO}

Introdução: O contexto da pandemia do novo coronavírus apontou para a importância de compreender a expansão geográfica desta doença, seus caminhos e efeitos, em especial nos territórios periféricos. Objetivo: Analisar e visibilizar os impactos da pandemia na porção da periferia oeste da Região Metropolitana do Rio de Janeiro, conhecida como Baixada Fluminense. Métodos (opcional): Baseado no método qualiquantitativo, utilizamos dados da Covid-19 disponibilizados pela Secretaria Estadual de Saúde do Rio de Janeiro nos primeiros meses da pandemia [março, abril e maio do ano de 2020], e realizamos uma análise da produção territorial da região de estudo para avaliar os efeitos e impactos do avanço desta doença. Resultados: Construção de mapas de calor da incidência da Covid-19 pelos bairros dos municípios da Baixada Fluminense que demonstraram as centralidades e os caminhos da doença pelos territórios periféricos, e produção de gráficos com o número de contaminados e novos casos em perspectiva comparada de parte da Região Metropolitana do Rio de Janeiro. Conclusão: A expansão geográfica do novo coronavírus tem revelado que as periferias metropolitanas são espaços privilegiados de contaminação, os dados apresentados demonstram um rápido crescimento do número de casos nessas áreas. Outrossim a maior incidência corresponde aos territórios que apresentam os caminhos de circulação metropolitana, maior centralidade no contexto dos municípios, grande adensamento populacional e maior vulnerabilidade econômica e social.

Palavras-chave: Pandemia - Covid-19 - Região Metropolitana - Rio de Janeiro Baixada Fluminense.

\begin{abstract}
Introduction: The context of the pandemic of the new coronavirus pointed to the importance of understanding the geographical expansion of this disease, its pathways, and effects, especially in peripheral territories. Objective: To analyze and visualize the impacts of the pandemic on the western periphery of the Metropolitan Area of Rio de Janeiro, known as Baixada Fluminense. Methods: Based on the qualitative and quantitative method, we used Covid-19 data provided by the State Department of Health of Rio de Janeiro in the first months of the pandemic [March, April and May of 2020], and performed an analysis of the territorial production of the study region to evaluate the effects and impacts of the advance of this disease. Results: Construction of heat maps of the incidence of Covid-19 by the neighborhoods of the municipalities of Baixada Fluminense that demonstrated the centralities and pathways of the disease through the peripheral territories, and production of graphs with the number of contaminated and new cases in a comparative perspective of part of the Metropolitan Area of Rio de Janeiro. Conclusion: The geographical expansion of the new coronavirus has revealed that the metropolitan peripheries are privileged spaces of contamination, the data presented show a rapid growth in the number of cases in these areas. Moreover, the highest incidence corresponds to the territories that present the traffic lane of metropolitan, greater centrality in the context of the municipalities, great population density and greater economic and social vulnerability.
\end{abstract}

Keywords: Pandemia - Covid-19 - Metropolitan Region - Rio de Janeiro - Baixada Fluminense.

\section{André Santos da Rocha ${ }^{a}$ \\ Dustavo Mota de Sousa ${ }^{a}$ \\ Alexandre Fortes ${ }^{a}$ \\ Deandro Dias de Oliveira ${ }^{a}$}

a Universidade Federal Rural do Rio de Janeiro (UFRRJ), Seropédica, RJ, Brasil

DOI: $10.12957 /$ geouerj.2021.51431

Correspondência:

asrgeo@gmail.com>

Recebido em: 01 jun. 2020

Aceito em: 03 nov. 2020

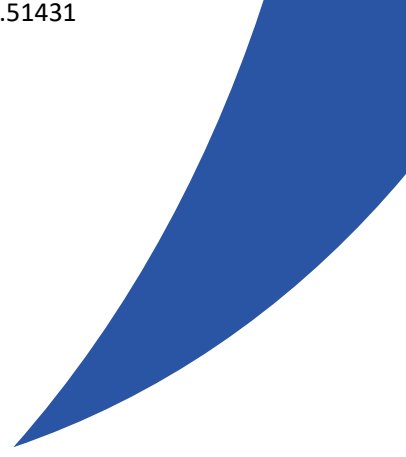




\section{RESUMEN}

Introducción: El contexto de la pandemia del nuevo coronavirus señaló la importancia de comprender la expansión geográfica de esta enfermedad, sus caminos y efectos, especialmente en los territorios periféricos. Objetivo: Analizar y visualizar los impactos de la pandemia en la periferia occidental de la Región Metropolitana de Río de Janeiro, conocida como Baixada Fluminense. Métodos: Sobre la base del método Cuali-cuantificativo, utilizamos datos de Covid-19 disponibles por el Departamento de Salud del Estado de Río de Janeiro en los primeros meses de la pandemia [marzo, abril y mayo de 2020], y realizamos un análisis de la producción territorial de la región del estudio para evaluar los efectos e impactos del avance de esta enfermedad. Resultados: Construcción de mapas de calor de la incidencia de Covid-19 por los barrios de los municipios de Baixada Fluminense que demostraron las centralidades y caminos de la enfermedad a través de los territorios periféricos, y la producción de gráficos con el número de casos contaminados y nuevos en una perspectiva comparativa de parte de la Región Metropolitana de Río de Janeiro. Conclusión: La expansión geográfica del nuevo coronavirus ha revelado que las periferias metropolitanas son espacios privilegiados de contaminación, los datos presentados muestran un rápido crecimiento en el número de casos en estas áreas. Además, la mayor incidencia corresponde a los territorios que presentan los caminos de circulación metropolitana, mayor centralidad en el contexto de los municipios, gran densidad de población y mayor vulnerabilidad económica y social.

Palabras clave: Pandemia - Covid-19 - Región Metropolitana - Río de Janeiro Baixada Fluminense. 


\section{INTRODUÇÃO}

Desde março de 2020, quando foi exposto o quadro de pandemia da Covid-19 pela OMS (Organização Mundial da Saúde), medidas de contenção do espraiamento da doença passaram a ser tomadas em todo o mundo. Na academia, perante a dimensão e possíveis impactos do avanço da doença, emergiram diversas reflexões. Numa era de globalidade virótica, não há dúvidas que a avaliar a dimensão geográfica da Covid-19 é também refletir sobre seus desdobramentos geoeconômicos e geopolíticos.

Artigos, dossiês e livros foram editados começam a ser publicados sobre o tema. No campo da geografia, as partes I e II do "Dossiê Coronavírus" publicado por Espaço e Economia: Revista Brasileira de Geografia Econômica1, o dossiê "Geopolítica da pandemia do Covid-19", publicado por Geopolítica (s) - Revista de estudos sobre espaço e poder ${ }^{2}$, da Universidade de Madri e obras como Coronavírus e a luta de classes ${ }^{3}$ (Terra sem Amos: Brasil, 2020), com textos dos geógrafos Mike Davis e David Harvey, revelam que o debate, como não poderia deixar de ser, somente se inicia.

O tratamento da origem e difusão do novo coronavírus (SARS-CoV-2) em escala mundial (CASTILHO, 2020); os impactos sobre os sistemas de saúde público e privado, as informações sobre a necessidade de quarentena e adoção de lockdown em alguns países (DAVIS, 2020; ROCHA, 2020; MONTERNELY, 2020; ZAAR \& GARCIA ÁVILA, 2020; FARIAS, 2020); os desafios políticos institucionais e diplomáticos oriundos da pandemia (AGNEW, 2020; IBAÑEZ, 2020; LOYER \& GIBLIN, 2020); a crítica aos modelos econômico-produtivos atual frente ao enfrentamento da pandemia; se tornaram temas recorrentes (HARVEY, 2020; ZIZEK, 2020, SANTOS SOUZA, 2020) e tendem a ser explorados detalhadamente em trabalhos futuros. Muitos artigos alertaram sobre a grande temeridade referente ao avanço da pandemia nos Países do Sul (MONIÉ, 2020; RíOS SIERRA, 2020), apresentando previsões pouco otimistas quanto a capacidade de resistência destes países perante à doença, ciente das condições socioespaciais de parte significativa de seus habitantes, especialmente das populações que vivem nas periferias metropolitanas (BARBOSA, 2020; OLIVEIRA et. al., 2020).

Desde março de 2020, o Brasil vive a transmissão comunitária do vírus. Num país com graves diferenças sociais e profunda insensibilidade política para com as populações periferizadas, tornou-se ponto-pacífico o prognóstico de avanço da doença para além dos grandes centros urbanos. Havia nos esforços analíticos a certeza de que a geografia contida na produção dos espaços periféricos (WACQUANT, 2007) e de penalização dos pobres (WACQUANT, 2009) indicava a imediata necessidade de aplicação de políticas públicas e sistemas inteligentes de gestão da crise humanitária que se avizinhava. Mas como as medidas políticas são

\footnotetext{
1 Disponível em: https://journals.openedition.org/espacoeconomia/10071 e https://journals.openedition.org/espacoeconomia/11397. Acesso em: 27 de maio de 2020.

2 Disponível em: https://revistas.ucm.es/index.php/GEOP/issue/view/3602. Acesso em: 27 de maio de 2020

${ }^{3}$ DAVIS, Mike et al. Coronavírus e a luta de classes. Terra sem Amos: Brasil, 2020. Disponível em:

https://terrasemamos.files.wordpress.com/2020/03/coronavc3adrus-e-a-luta-de-classes-tsa.pdf. Acesso em: 27 de maio de 2020.
} 
espacialmente assimétricas e antidemocráticos, e, se num primeiro momento o vírus atingiu uma população de condições mais abastadas, capazes de realizar viagens internacionais ou manter contato com possíveis sujeitos globalizados, atualmente ela atinge majoritariamente a população mais pobre e trabalhadora das periferias urbanas.

A importância de análises como a proposta neste artigo se justifica pelo pela expansão espacial combinada com a ampliação dos números de contaminados e óbitos nas áreas das periferias urbanas. $\mathrm{O}$ que se prepõe é o olhar da periferia metropolitana do Rio de Janeiro, estado que nos meses de março, abril e maio de 2020 se consolidou como o segundo em números da doença no Brasil, atrás apenas de São Paulo. Neste momento, mesmo com contaminados em todas os municípios do estado, somente a capital e as treze cidades que compõem a Baixada Fluminense aglutinam mais de $80 \%$ dos casos da Covid-19 de todo o estado do Rio de Janeiro.

Desse modo, o presente artigo apresenta uma análise da expansão geográfica da Covid-19 na Baixada Fluminense, entendida como periferia imediata da cidade do Rio de Janeiro. O objetivo é apresentar, no cenário de pandemia da Covid-19, a dinâmica interna dos municípios da Baixada com base nos dados disponibilizados pela Secretaria Estadual de Saúde do Rio de Janeiro, nos meses de março, abril e maio de 2020.

Os esforços aqui contidos são resultado do trabalho cotidiano de monitoramento de diferentes laboratórios da Universidade Federal Rural do Rio de Janeiro (UFRRJ), por meio de: [1] levantamento, acompanhamento e tabulação da evolução dos dados; [2] construção de diferentes mapas - metropolitanos, estaduais, rodoviários, em formato anamorfose etc. - com o número de contaminados, curados, óbitos e demonstrando as centralidades e os caminhos da Covid-19; [3] redação de uma série de notas de pesquisa disponibilizadas na plataforma do Open-Lab do Programa de Pós-Graduação Interdisciplinar em Humanidades Digitais da UFRRJ, que acabaram fomentando reportagens na grande mídia e especialmente contribuindo com relatório de posicionamento da Fundação Oswaldo Cruz (Fiocruz) perante a pandemia da Covid-194.

Para atingir tal escopo, estruturamos o artigo em mais quatro partes. Na primeira parte, apresentamos as notas teórico-metodológicas referentes à pesquisa, que consiste em definir a área de estudo, detalhar a metodologia da coleta e tratamento dos dados e de elaboração dos mapas e gráficos inseridos neste projeto de acompanhamento e difusão da Covid na Baixada Fluminense e Rio de Janeiro. Na segunda parte, apresentamos os resultados analíticos de acompanhamento da geografia da Covid-19 em seus caminhos pela

\footnotetext{
${ }_{4}^{4}$ FIOCRUZ. Presidência da Fiocruz. Posicionamento da Fundação Oswaldo Cruz (Fiocruz) -06/05/2020: a evolução da Covid-19 no Estado do Rio de Janeiro: desafios no enfrentamento da crise sanitária e humanitária relacionada à pandemia. Rio de Janeiro, 2020. 19 p. Disponível em: https://www.arca.fiocruz.br/bitstream/icict/41174/2/relatorio_distanciamentosocial.pdf. Acesso em 27 de maio de 2020.
} 
Baixada Fluminense, interpretando espacialmente sua dispersão entre os meses de março e abril. Na terceira parte, realizamos uma exame qualitativo sobre o avanço da Covid-19 no contexto da periferia metropolitana, apontando as ações para o enfrentamento da doença pelos poderes públicos municipais e os descaminhos do combate frente ao "apagão" de dados existentes, Por fim, à guisa de conclusão, realizamos um balanço dos apontamentos da pesquisa e apresentamos indicativos que possam subsidiar políticas públicas nesses territórios periféricos.

Notas teórico-metodológicas da pesquisa: definição da região, coleta e tratamento dos dados

É consenso na literatura da geografia urbana do Estado do Rio de Janeiro a compreensão da Baixada Fluminense como periferia da cidade do Rio de Janeiro (ABREU, 2013). Seja histórica e concretamente, seja pela sua representação hegemônica, a Baixada carrega o signo de área violenta, marcada por péssimos indicadores sociais. Não é por outro motivo que o geógrafo Roberto Lobato Corrêa (2005, p.160), fundamental nos estudos de hierarquia urbana, usa a Baixada como exemplo explicativo sobre periferia metropolitana, pois a compreende como "o lugar da existência de parcela ponderável das camadas populares".

Entendemos que a Baixada está associada aquilo que Milton Santos (2009) denominou de uma periferia verdadeiramente periférica: geometricamente distante do processo de centro, esta periferia abre conteúdos e especificidades que a colocam em seu teor, derivado de um processo maior de urbanização típica da periferia mundial. Trata-se de uma periferia "seletiva", incluída de forma segregada nos processos de modernização e inclusão-exclusão e reprodutora de desenvolvimento desigual.

A Baixada Fluminense corresponde à área que foi incorporada pela célula urbana do Rio de Janeiro, nucleada pela cidade de Nova Iguaçu (SEGADA SOARES, 1962), que viveu um processo acelerado de urbanização fomentado por meio do declínio da atividade agrícola. Este declínio permitiu um novo uso dos espaços através de loteamentos com grande ausência de infraestrutura (GEIGER \& SANTOS, 1955; SIMÕES, 2007; SOUZA, 2007), junto à consolidação de graves contrastes sociais e interdependência com a cidade do Rio de janeiro. Mesmo com os recentes processos de transformação econômica e produtiva (LAGO, 2007; OLIVEIRA, 2015), as contradições internas permanecem, quando não se aprofundam (ROCHA, 2019).

A despeito da multiplicidade de composições territoriais e diferentes delineamento de limites, trabalhamos com o recorte regional de Baixada Fluminense baseado no conjunto de trezes municípios que estão associados por filiações políticas e institucionais e guardam similitudes nos processos de urbanização e possibilidades de interconexão. Nomeadamente, esta Baixada Política (SIMÕES, 2007) é composta pelos municípios de Nova Iguaçu, Duque de Caxias, Belford Roxo, Queimados, Japeri, Paracambi, Magé, Guapimirim, Nilópolis, São João de Meriti, Mesquita, Seropédica e Itaguaí. A própria Baixada Fluminense também apresenta 
diferenças, especialmente entre a baixada histórica, de origem iguaçuana e com urbanização mais densificada - que passa por uma reestruturação urbano-econômica (ROCHA, 2015), e a porção do extremo oeste metropolitano formado pelas cidades de Seropédica, Itaguaí, Paracambi, Japeri e Queimados (OLIVEIRA, 2014), além de Magé e Guapimirim, que compõem uma transição Baixada-Leste Metropolitano (OLIVEIRA, 2020) (Figura 1)

Figura 1. Região Metropolitana do Rio de Janeiro, com destaque para a Baixada Fluminense e suas "sub-regiões"

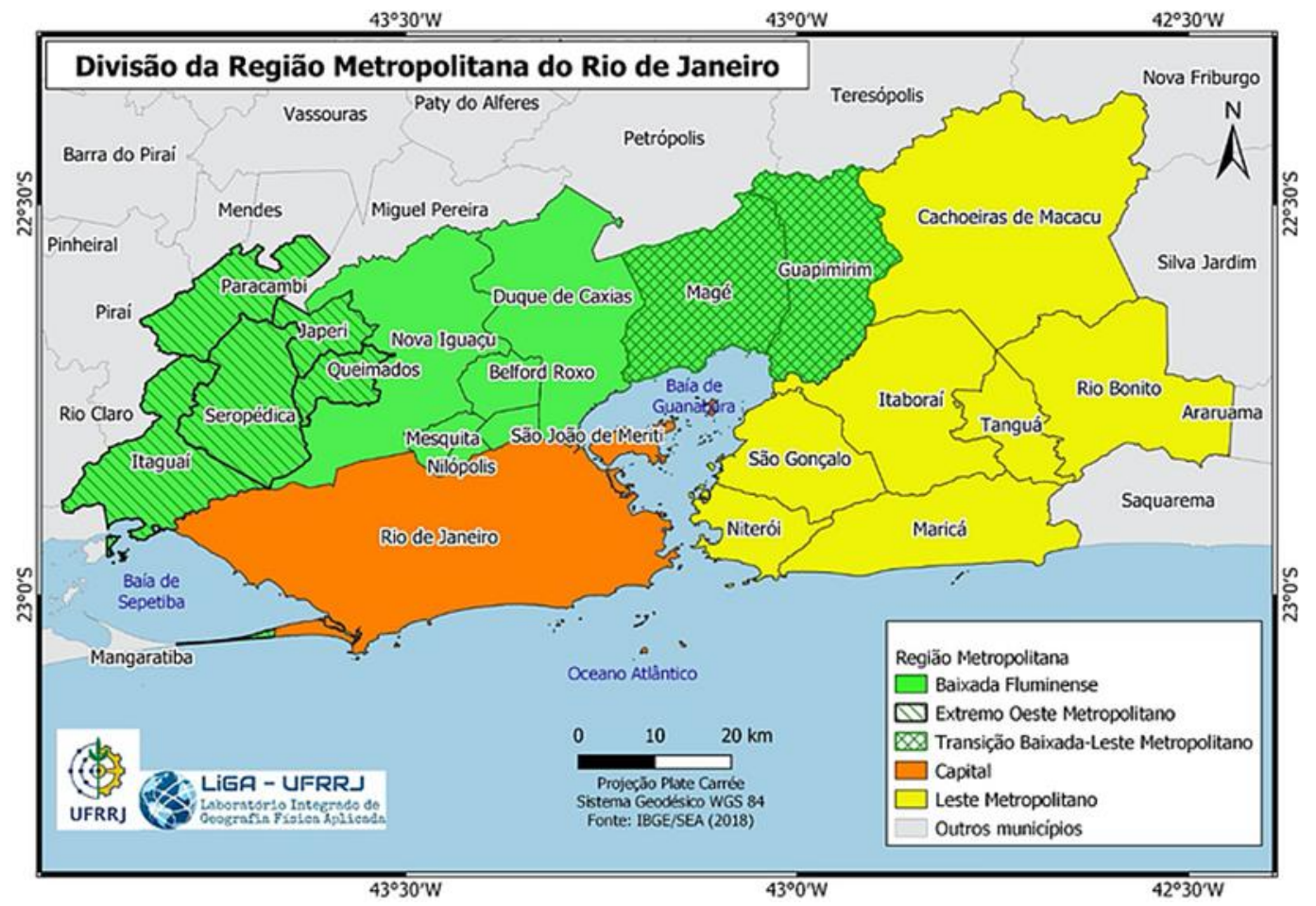

Organização e Elaboração: Laboratório Integrado de Geografia Física Aplicada (LiGA-UFRRJ) e Laboratório de Geografia Econômica e Política (LAGEP-UFRRJ) [2020]. Disponível em: https://www.ppgihdopen-lab.com/post/para-al\%C3\%A9m-da-capital. Acesso em: 27 de maio de 2020.

É justamente a partir deste recorte espacial, formado por treze municípios metropolitanos do Rio de Janeiro, que objetivamos pensar acerca dos impactos sociais decorrentes do avanço da Covid-19. Nós entramos em um momento em que se exigem políticas articuladas de combate ao novo coronavírus e, destarte, a intepretação dos dados do número de infectados, quando analisados espacialmente, permitem compreender o conteúdo social dois espaços de contaminação e eixos de difusão.

Lamentavelmente, há uma sentida ausência de dados espaciais de localização por bairro, gênero, raça, entre outros, que revela um verdadeiro "apagão de informações", devido à combinação da velocidade de 
propagação da doença com a pequena quantidade de testes realizados pelas autoridades de saúde no país. Se entendemos que o levantamento, tratamento e divulgação de dados e informações confiáveis são capazes de subsidiar a constituição de políticas públicas profícuas, não é possível desconsiderar as constantes lacunas, falhas e desinformações das esferas públicas da saúde, e tampouco deixar de lembrar que a diminuta quantidade de testes exprime, certamente, que os números divulgados estão muito aquém da realidade da pandemia. Todavia, tais análises potencializam ações no espaço geográfico, particularmente no trato dos espaços periféricos sempre tão suscetíveis às mazelas sociais. Nesta verdadeira inteligência territorial, tornase premissa reconhecer as dinâmicas singulares dos lugares, com o intento de avaliar a efetividade das ações e gestar estratégias.

Foi com base nesta premissa que estamos realizando um levantamento sistemático de informações espaciais sobre a Covid-19 na Baixada Fluminense. Referenciados no registro dos bairros onde foram assinalados nos relatórios os indivíduos infectados pelo novo coronavírus, construímos um mapa da incidência da Covid-19 na Baixada Fluminense. Este mapeamento apresenta como resultado a indicação dos locais de concentração do número de casos, com a observação do seu raio de amplitude a partir das aglomerações. A metodologia de construção do mapa de incidência considerou os dados referentes aos indivíduos contaminados pela Covid-19, disponibilizados pela Secretaria Estadual de Saúde do Rio de Janeiro (SES-RJ, 2020), no período entre 11 de março até 21 de abril deste ano de 2020 (Figura 2) que compreende as semanas epidemiológicas $12,13,14,15$ e $16^{5}$. O período do estudo se refere, respectivamente, a data da primeira contaminação dos indivíduos pelo novo coronavírus na Baixada Fluminense com a identificação dos bairros até o término desse dado de localização, hoje limitado apenas a dados gerais por município.

A edição dos dados da SES (2020) foi realizada pela equipe do LAGEP-UFRRJ ${ }^{6}$ visando a construção de uma tabela com a identificação e contabilização dos casos confirmados por bairro. Os dados espaciais vetoriais dos limites dos bairros foram adquiridos a partir do Portal do Ministério Público do Estado do Rio de Janeiro (MPRJ) - MPRJ In loco (http://apps.mprj.mp.br/sistema/inloco/). Esses dados vetoriais estão disponíveis em formato shape e abrangem os bairros da Região Metropolitana em polígonos que são baseados nas leis municipais e Planos Diretores.

\footnotetext{
${ }^{5}$ As semanas epidemiológicas, é uma convenção internacional, que correspondem aos dias entre domingo e sábado que marcam a semana de transmissão de doenças. O calendário total pode ser acessado em < http://www.portalsinan.saude.gov.br/calendarioepidemiologico>.

${ }^{6}$ Os dados foram organizados pela equipe de apoio de dados sobre o Covid-19 do Laboratório de Geografia Econômica e Política da UFRRJ (LAGEP-UFRRJ), composta por: Juliana Borges Ferreira, Jhonatan Bento da Silva, Maria Fernanda Gomes Arcanjo e Pedro Ferreira Duarte Neto, todos discentes do curso de Relações Internacionais da UFRRJ; Bruno Luís de Souza Felix dos Santos e Camila Gonçalves dos Santos, alunos do curso de Geografia, campus Seropédica, UFRRJ; e Vanessa Ferreira Andrade, discente do curso de Geografia da UERJ, campus Maracanã. Agradecemos imensamente pelo trabalho realizado.
} 
Figura 2. Incidência de casos da Covid-19 na Baixada Fluminense. Organização e Elaboração: Laboratório de Geografia Econômica e Política (LAGEP-UFRRJ) e Laboratório Integrado de Geografia Física Aplicada (LiGA-UFRRJ) [2020]. Disponível em: https://www.ppgihd-open-lab.com/post/os-caminhos-da-Covid-19-na-baixada-fluminense. Acesso em: 27 de maio de 2020.

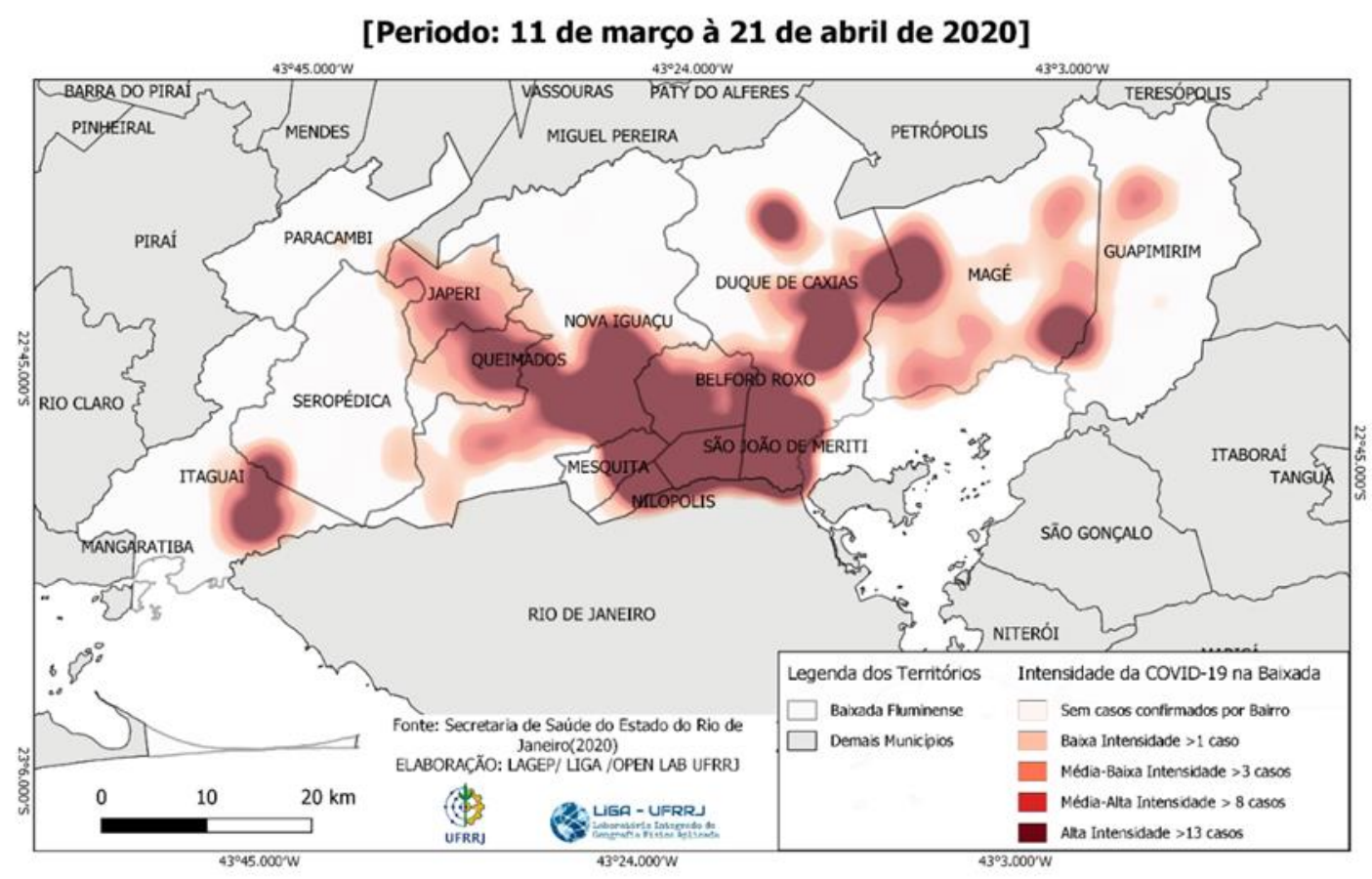

Com vistas a observar o fenômeno da Covid-19 baseado no uso de estatística espacial por meio da técnica de Kernel, foram gerados os centros dos polígonos de bairro e a validação e ajuste da localização dentro de ambientes Google Earth e QGIS 3.10 para os procedimentos de inserção dos dados na tabela. A estimativa Kernel, realizada no QGIS 3.10, é uma técnica de interpolação exploratória que gera uma superfície de densidade para a identificação visual de "áreas quentes" (SANTOS, SOUZA, 2007).

Essa metodologia foi aplicada para construção do referido mapa 2 e dos mapas 3 e 4 . Se tratando de um primeiro período do ciclo de contaminação e expansão do novo coronavírus, a partir das cinco primeiras semanas epidemiológicas registradas para o recorte especial selecionado, foram estabelecidos cinco intervalos para avaliar essa intensidade de incidência, definidos pela estimativa de kernel através da programação do QGIS 3.10. Sendo: alta intensidade - configurando bairros que contabilizaram mais de 13 casos, sendo que o máximo encontrado foi de 23 casos; média-alta intensidade com os registros entre 5 a 12 casos; média-baixa intensidade com os bairros que contabilizaram 3 e 4 casos; baixa intensidade que representa os bairros com 1 caso confirmado; e sem casos confirmados por bairro - que se refere aqueles bairros que no período não foram identificados oficialmente registros de contaminação.

As classes que possuem casos de COVID-19 são identificadas pelas áreas quentes, e intensificam-se com as variações de tons avermelhados seguindo o aumento dos registros. Elas indicam uma concentração de 
eventos que representam, de alguma forma, uma superfície contínua das ocorrências que fazem parte do grupo de dados que está sendo observado - números de casos de contaminação pelo novo coronavírus, conforme as informações do mapa 2, que detalharemos a seguir.

\section{Os caminhos da Covid-19 na periferia e seu espraiamento na Baixada Fluminense}

A análise da figura 2 permite notar a alta incidência na Baixada Iguaçuana, área que é fortemente urbanizada e de maior coesão quanto a estrutura econômica social do que se conhece popularmente como a representação hegemônica da Baixada Fluminense. Ela compreende a espacialidade do contínuo-denso do Covid-19, marcada pela cor vermelha mais escura, na extensão territorial entre os municípios de Nova Iguaçu, Belford Roxo, Mesquita, Nilópolis, São João de Meriti e Duque de Caxias. Destacamos que quatro (4) destes municípios - São João de Meriti, Mesquita, Nilópolis e Belford Roxo - foram enquadrados em recente estudo da Fundação Perseu Abramo entre os trinta municípios mais vulneráveis ao avanço da doença no Brasil7. Entre os fatores elencados que transformam esses municípios em locais com extrema vulnerabilidade para o avanço da doença estão a alta densidade demográfica, as fragilidades dos vínculos e remunerações do trabalho enfrentadas por grande parte da população, as condições nas estruturas sanitárias e a parca disponibilidade de equipamentos de saúde.

Além disso, a Baixada Fluminense possui graves problemas estruturais no que tange a assistência aos serviços urbanos básicos, que envolvem a ineficiência do saneamento básico (água e esgoto), as ocupações irregulares, a deficiência nos equipamentos de saúde, entre muitos outros. A posição geográfica, contígua à capital do Rio de Janeiro e recortada por importantes vias de circulação e eixos ferroviários destinados à locomoção de massas trabalhadoras e de mercadorias, coloca a Baixada Fluminense no front territorial da expansão da Covid-19 no estado. Assim, torna-se possível perceber duas grandes vetorizações regionais com base nos eixos das principais rodovias federais que cortam a Baixada: a BR-040 (Avenida Washington Luiz) e a BR-116 (Avenida Presidente Dutra).

A primeira vetorização revela a dispersão da doença no interior do município de Duque de Caxias e seu espraiamento em direção a Magé e Guapimirim, e, a seguir, para a Região Serrana, tendo Petrópolis como local de convergência ${ }^{8}$. (ver figura 3 ).

\footnotetext{
${ }^{7}$ Consultar: https://www.ppgihd-open-lab.com/post/s\%C3\%A3o-jo\%C3\%A3o-de-meriti-\%C3\%A9-a-cidade-brasileira-maisvulner\%C3\%A1vel-ao-alastramento-do-coronav\%C3\%ADrus. Acesso em: 04 de maio de 2020.

8 O historicamente complexo delineamento da Região Metropolitana do Rio de Janeiro (RMRJ), bem como a nomeação, delimitação e entendimento das demais regiões do estado, oferecem desafios da compreensão do fenômeno metropolitano fluminense em sua plenitude. Petrópolis, claramente cidade serrana, foi recentemente incluída como pertencente à RMRJ. Nos reservaremos a tratar como noção de espaço e região metropolitana a área de influência da economia urbana real a partir da capital, a cidade do Rio de Janeiro.
} 
Figura 3. mapa da Incidência de casos de Covid-19 em Duque de Caxias e Arredores. Organização e Elaboração: Laboratório de Geografia Política e Econômica e Laboratório Integrado de Geografia Física da UFRRJ. Fonte: Secretaria de Saúde do Estado do Rio de Janeiro.

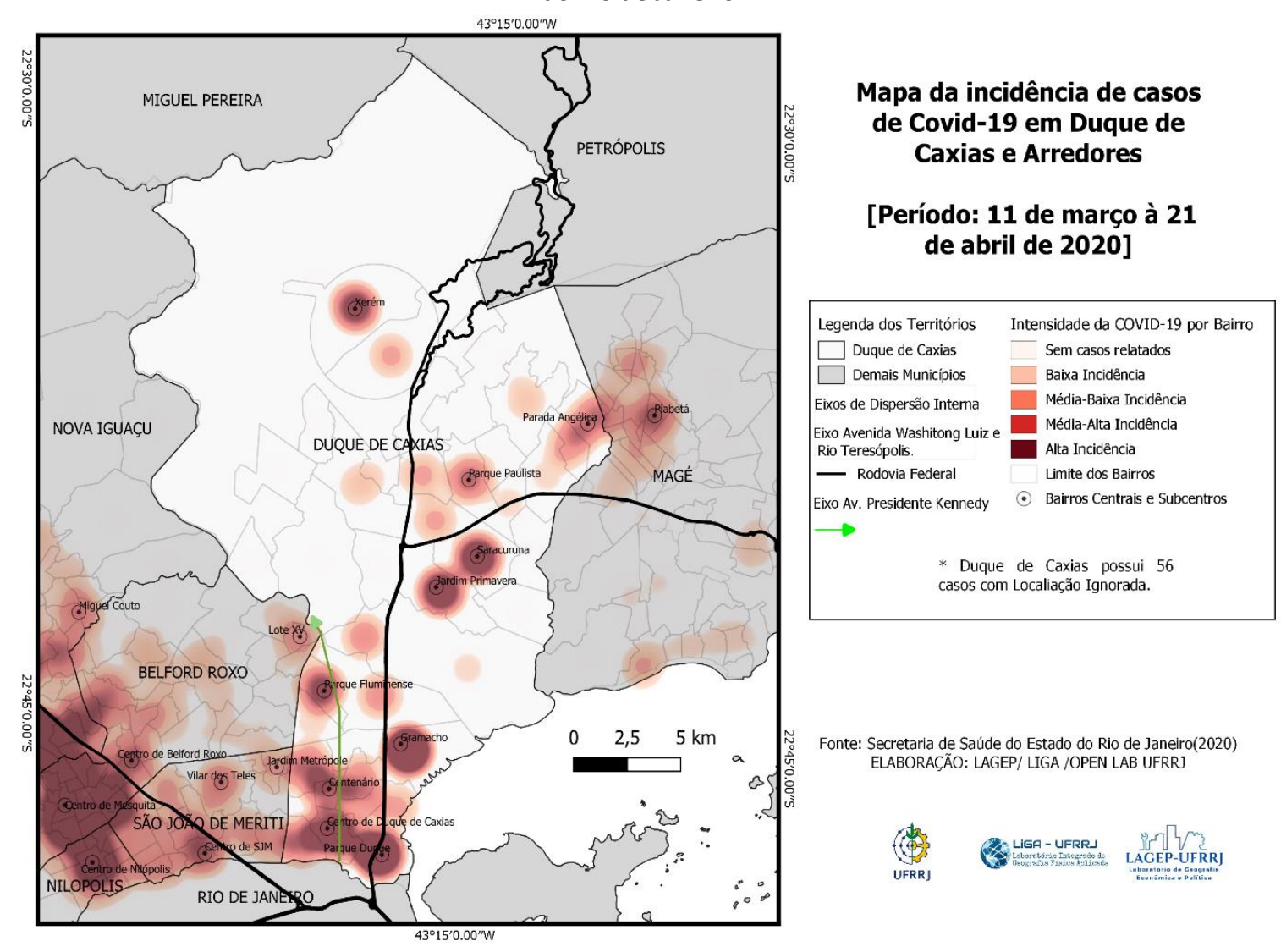

No caso de Duque de Caxias, destacam-se as áreas de conurbação com os municípios de São João de Meriti, Belford Roxo e Rio de Janeiro - neste último, por meio dos bairros Brás de Pina e Cordovil. Toda esta

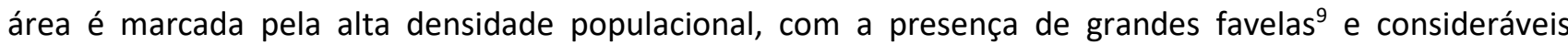
centralidades econômicas locais. É por isso que bairros como Parque Duque (17 Casos) e Gramacho (19 casos $)^{10}$ foram os mais afetados com casos da doença em toda a Baixada, estando apenas atrás do centro de Nova Iguaçu (23 casos) e do Centro de São João de Meriti (19 casos). Assim, a análise da cidade Duque de Caxias expressa dois eixos de expansão da Covid-19: [a] o eixo da BR-040, que envolve os bairros de Jardim Primavera e Saracuruna, ambas com 12 casos, e Xerém (10 casos), e também o [b] o eixo Avenida Presidente

\footnotetext{
${ }^{9}$ Diante da grande dificuldade de definição das áreas das favelas pelas autoridades locais, tratando-as como aglomerados subnormais, somada à percepção dos moradores que faz seus próprios critérios de "classificação", podemos citar neste trecho, pelo menos três complexos na área: Mangueirinha, Favela do Lixão e Favela Beira-Mar.

${ }^{10}$ Devido a imprecisão das informações sobre bairros da SES (2020), muitas vezes mencionando alguns sub-bairros, ou áreas com nomes de ruas locais, e mesmo bairros não existentes; e pela ausência de base cartográfica que informasse a geometria destas localidades, aproximamos algumas localidades em bairros reconhecidos com base na localização georreferenciada do Google Earth . Daí, a grande contabilização geral dos Bairros Gramacho, inserido informações de Vila Leopoldina (11 casos), Jardim Gramacho (4 casos); e do Bairro Parque Duque que agregou dados de Parque das Missões (3 casos), Vila Guanabara (3 casos), Parque Beira mar (2 casos); Parque Boa Vista (1 caso), Vila Oito de Maio (1 caso) e Vila Operária (1 caso).
} 
Kennedy, que tem como centralidades de casos o Centro de Duque de Caxias com 8 casos, Parque Fluminense (9 casos), Centenário (7 casos) e São Bento (5 casos).

A outra vetorização esboça outro caminho de interiorização da Covid-19 a partir da concentração de casos em Nova Iguaçu que se espraia pelo seu entorno no contínuo-denso da Covid-19 na Baixada. Esta vetorização tem a rodovia Presidente Dutra (BR-116) como um eixo de expansão, conforme mapa a seguir (Figura 4).

Figura 4. Incidência de Casos de Covid-19 em Nova Iguaçu e arredores. Organização e Elaboração: Laboratório de Geografia Econômica e Política (LAGEP-UFRRJ) e Laboratório Integrado de Geografia Física Aplicada (LiGA-UFRRJ) [2020]. Disponível em: https://www.ppgihd-openlab.com/post/os-caminhos-da-Covid-19-na-baixada-fluminense. Acesso em: 27 de maio de 2020.

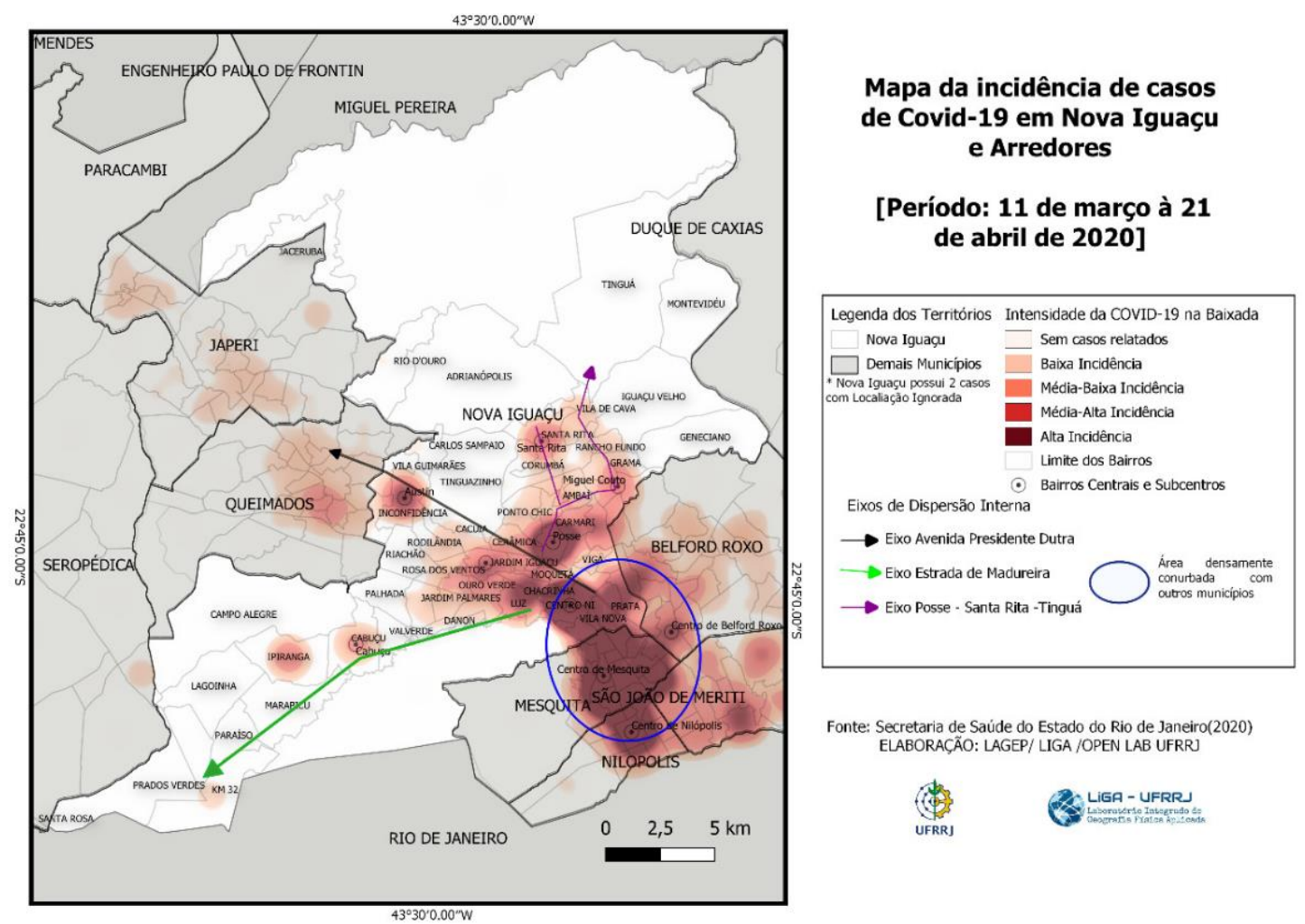

Essa área apresenta o Centro de Nova Iguaçu como centralidade pelo número de casos (23), que se propaga internamente para os centros e localidades importantes de municípios vizinhos que são relativamente próximos: Centro de São João (19 casos), Centro de Nilópolis (9 casos), Centro de Mesquita (7 casos) e Edson Passos (10 casos) - esses três ligados pela linha férrea - e o Centro de Belford Roxo (7 casos). Ainda há a evidência da interiorização pelos bairros internos de Nova Iguaçu, revelando a relação centroperiferia e marcando o avanço interno do novo coronavírus. Assim, podemos verificar em Nova Iguaçu três eixos de expansão: [a] o eixo da Rodovia Presidente Dutra que é, ao mesmo tempo, de interiorização pelos 
demais bairros de Nova Iguaçu, destacando-se Comendador Soares (6 casos) e Austin (8 casos), e de expansão para outros municípios, em especial no que se refere ao avanço em direção ao Médio Vale Paraíba Fluminense, impactando cidades como Queimados, Japeri e Paracambi; [b] o eixo da Estrada de Madureira, que atinge o Km 32, no limite entre as cidades de Seropédica e Rio de Janeiro, atravessando bairros como Luz (6 casos), Ipiranga ( 5 casos) e Cabuçu (4 casos); [c] o eixo Posse-Santa Rita-Tinguá, que se revela um eixo interno de grande intensidade, que passa pelo bairro Posse (com 13 casos), e perpassa por inúmeras vias secundárias de circulação da RJ-111, ligando bairros como Miguel Couto (5 casos) e Vila de Cava ( 3 casos), e a partir de linhas auxiliares chega até Santa Rita (6 casos).

É possível incluir como desdobramento deste último eixo de dispersão os casos do município de Seropédica, que acaba tendo sua influência de contaminação, pelos contatos com Itaguaí, cujos veios de contato - Reta de Piranema (RJ-099) e Arco Rodoviário Metropolitano (BR-493), e com a cidade do Rio de Janeiro, pelo trasbordamento dos casos existente na Zona Oeste do município, que permite um avanço via Km 32, na travessia da BR-456 (antiga Estrada Rio-São Paulo), que corta o municípios de Seropédica e liga a Avenida Brasil (BR-101) com a Avenida Presidente Dutra (BR-116). Seropédica é considerada uma importante centralidade logística, pelos inúmeros contatos estradais, pela proximidade e papel de estabelecido de retroporto de Itaguaí, por ser sede de galpões e condomínios de armazenagem e logística e ainda por ter atraído inúmeros investimentos produtivo-industriais nos últimos anos. A favor de Seropédica, destacamos a proficuidade das ações da Universidade Federal Rural do Rio de Janeiro, que permitiu evitar que o campussede, com seus milhares de docentes, discente e servidores técnicos-administrativos, se tornasse um privilegiado espaço de contaminação, bem como tem realizado uma série de ações para com seu entorno, da produção de máscaras e álcool $70^{\circ}$ até a realização de atividades de pesquisa, extensão e informação sobre a pandemia ${ }^{11}$.

Por fim, os dados da Covid-19 que foram disponibilizados pela SES, editados e transformados nos mapas apresentados, poderiam ganhar outras indicações. Porém, devido aos casos de localização ignorada, muitas tendências internas não puderam ser compreendidas na íntegra. Por exemplo, nas cidades de Duque de Caxias e São João de Meriti, foram ignorados o bairro de, respetivamente, 69 e 19 casos, números consideráveis para exercícios analíticos desta natureza.

\footnotetext{
11 Torna-se oportuno destacar que a UFRRJ tem realizado ações que fomentem a ação solidária institucional, que envolvem a produção de álcool em gel, máscaras e produção de cestas de alimentos orgânicos. Para além das ações institucionais, uma infinidade de ações solidárias realizadas por professores e demais servidores, alunos de diferentes modalidades e cursos e outros membros da comunidade acadêmica e local, em grande parte de maneira discreta e sem grande alarde, tem proporcionado alimentos, itens básicos de higiene e outros artigos que permitem levar conforto e esperança aos mais necessitados.
} 
Uma análise social, econômica e demográfica da Covid-19 na Baixada.

O Brasil, dolorosamente, apresenta atualmente uma tendência de agravamento da pandemia, e o sistema de saúde, em suas redes públicas e particulares, quando não já se apresentam colapsados, estão à beira da falência em várias cidades. O caráter continental do Brasil, marcado por profundas diferenças regionais, gera ritmos diferentes na difusão do vírus, assim como para as extremas desigualdades sociais que fragilizam a maioria da população no que diz respeito aos riscos de contaminação e evolução para quadros graves. Nesse sentido, o acompanhamento dos dados sobre diferentes áreas da Região Metropolitana do Rio de Janeiro gera motivos para preocupação adicional, e comparações entre a capital e a Baixada Fluminense se mostram necessárias. Os dados aqui apresentados foram coletados são reveladores das novas tendências da pandemia.

Vamos enfocar aqui apenas a relação entre duas dessas áreas. De um lado, a AP2, composta pela Zona Sul da capital do estado e a chamada Grande Tijuca. De outro, a grande área contínua de altíssima densidade de ocupação desordenada e precária formada pela AP3 da capital (Ramos, Penha, Inhaúma, Méier, Irajá, Ilha do Governador, Anchieta, Pavuna, Jacarezinho, Complexo do Alemão, Maré e Vigário Geral) e por seis municípios da Baixada Fluminense (Duque de Caxias, Nova Iguaçu, São João de Meriti, Belford Roxo, Nilópolis e Mesquita).

A AP2, que de acordo com o IBGE possuía em 2010 pouco mais de um milhão de habitantes, nas primeiras semanas após a pandemia atingir o Brasil, respondia pela grande concentração de casos e de óbitos no Rio de Janeiro. Inicialmente, tratavam-se de casos importados do exterior, que posteriormente deram origem a focos de transmissão comunitária. Apesar de conter muitas favelas, AP2 concentra o maior número de residências de classe média da região metropolitana, e possui uma excelente rede de serviços de saúde. Por outro lado, possui uma alta proporção de idosos, o que agrava a tendência a casos graves e óbitos.

A realidade socioeconômica da AP3 e dos municípios contíguos da Baixada Fluminense é profundamente diferente. Com cerca de cinco milhões e quatrocentos mil habitantes, essa área possui altos índices de tuberculose, internações por diarreia e de incidência de diversos outros problemas de saúde associados a condições sub-humanas de habitação e saneamento. A rede de saúde é absolutamente precária, e praticamente não existe articulação institucional das esferas de governo para planejar o enfrentamento a um desafio sanitário desta magnitude.

O sinal de que o epicentro da pandemia na região metropolitana se desloca pouco a pouco para essa área já são visíveis (ver: Figura 5). O número absoluto de casos, tanto na AP3 quanto na Baixada (incluindo também os sete municípios mais distantes da capital e com menos casos), já superou o da AP2, apesar de 
também continuar em ascensão nela, possivelmente atingindo agora mais as grandes favelas do que a classe média.

Figura 5. Covid-19: Casos confirmados nas cidades do Rio de Janeiro e na Baixada Fluminense. Organização: Alexandre Fortes. Disponível em: https://www.ppgihd-open-lab.com/post/covid-19-brasil-\%C3\%A9-a-bola-da-vez-e-periferia-do-rio-de-janeiroser\%C3\%A1-duramente-atingida. Acesso em 15 de maio de 2020.

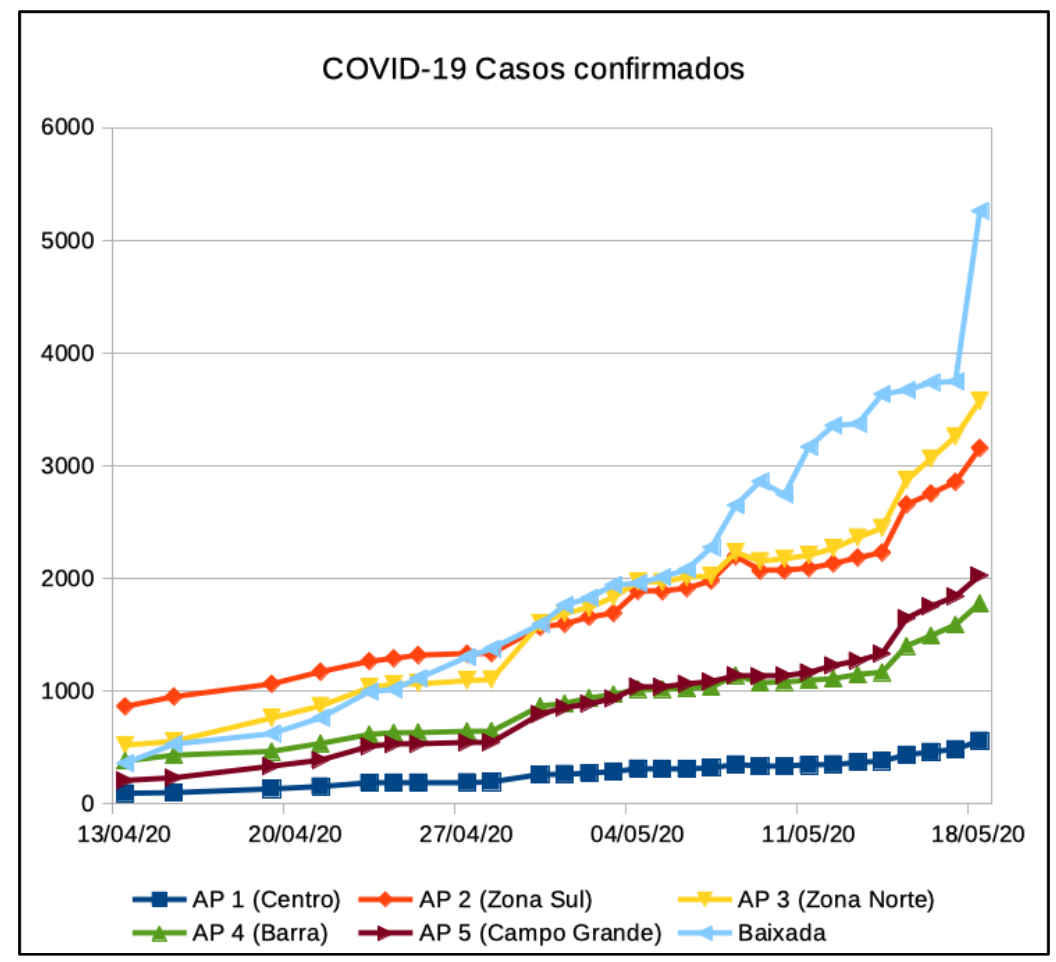

Em relação aos óbitos, a AP3 (293) já ultrapassou a AP2 (269), que está praticamente empatada com a Baixada (268). Na primeira semana de maio, os óbitos cresceram 75\% na Baixada, 62\% na AP3 e 56\% na AP2. Considerando-se que os óbitos refletem contaminações ocorridas há duas ou três semanas, a tendência é que essas diferenças nas taxas de crescimento entre as áreas tendam a se acentuar. Portanto, se até esta data a taxa de óbitos por milhão de habitantes na AP2 (267) ainda é muitíssimo superior à da AP3 (122) e mais de três vezes superior à dos seis municípios da Baixada citados acima (81), isso, novamente, só reforça a tendência de que o número de fatalidades venha a se multiplicar de forma acelerada nas áreas onde o impacto da pandemia é mais recente. Se o Brasil caminha para se tornar um dos epicentros da pandemia no mundo e nada indica que o pico da primeira onda esteja sequer próximo, a Baixada Fluminense também caminha para se tornar epicentro da pandemia no estado do Rio de Janeiro e tende a se tornar um dos principais centros do crescimento do número de casos e de óbitos no país. 
Essa tendência exige reforço às medidas de isolamento social, combinado com campanhas informativas, medidas de transferência de renda e assistências social, planejamento integrado das ações de saúde entre diferentes esferas de governo, mobilização solidária de todos os segmentos e atores sociais. Menosprezar a gravidade da situação pode ampliar em muito a escala do sofrimento humano que, infelizmente, já se faz presente à vida da maioria das famílias e das comunidades.

Agrupando os dados sobre casos novos por semana epidemiológica, percebemos que, enquanto no início de maio, o ritmo de crescimento da pandemia havia caído, o afrouxamento verificado posteriormente já se reflete numa retomada, em ritmo mais acelerado do que antes, do crescimento do número de casos, particularmente na Baixada, na AP3 (Zona Norte, exceto Grande Tijuca) e na AP5 (Bangu, Campo Grande, Santa Cruz, etc.). Já em 18 de maio, verificou-se uma explosão do número de casos confirmados na Baixada Fluminense, que saltaram de 3.747 para 5.259, um aumento de $40 \%$ em 24 horas. Esse salto concentrado em um único dia certamente se deve ao represamento de testes em processamento, mas indica também que a tendência de alta já verificada no período anterior estava subdimensionada até então (Ver: Figura 6).

Figura 6. Covid-19: Novos casos por Semana Epidemiológica nas cidades do Rio de Janeiro e na Baixada Fluminense. Organização: Alexandre Fortes. Disponível em: https://www.ppgihd-open-lab.com/post/covid-19-brasil-\%C3\%A9-a-bola-davez-e-periferia-do-rio-de-janeiro-ser\%C3\%A1-duramente-atingida. Acesso em 15 de maio de 2020.

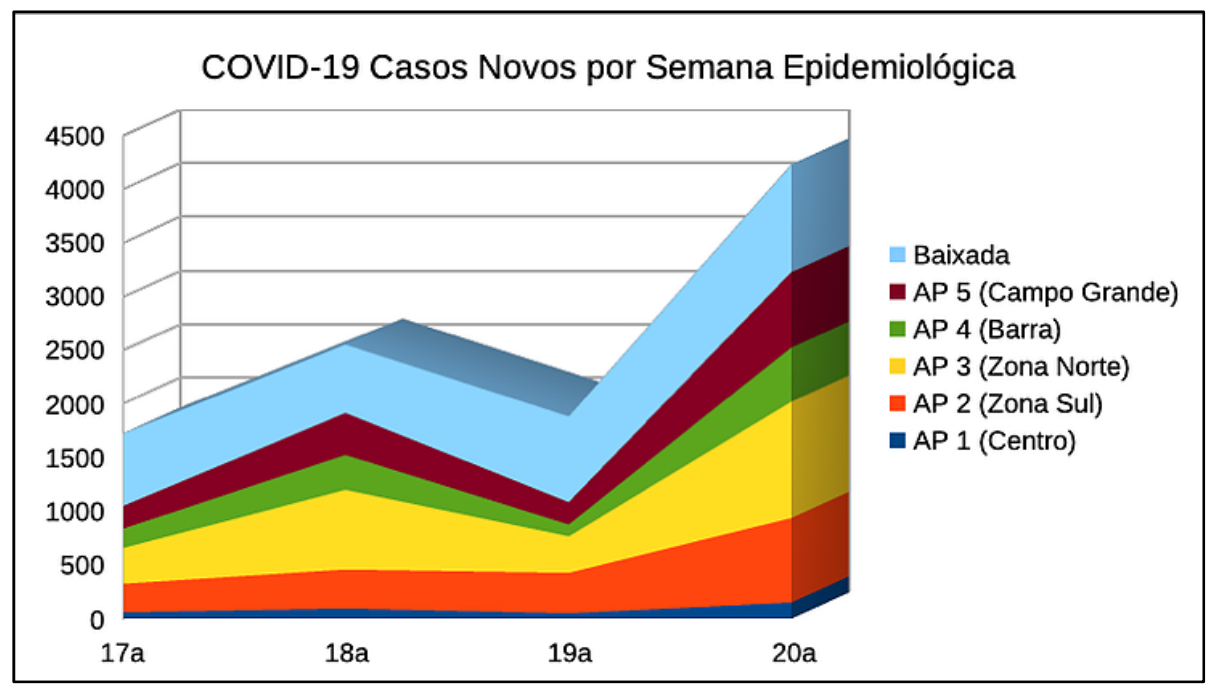

Em relação ao número de novos óbitos, as duas últimas semanas epidemiológicas já demonstram redução na AP2 (Zonal Sul e Tijuca), aumento expressivo na AP5 e na Baixada e, de forma ainda mais intensa, na AP3. É importante destacar que o crescimento do número de novos óbitos nessas áreas na 20a semana epidemiológica ainda reflete um aumento modesto no número de casos quinze dias atrás, o que indica um subsequente crescimento muito mais expressivo. 
Isso indica a migração do epicentro da pandemia na região metropolitana de áreas com uma qualidade de vida que pode ser sintetizada no Índice de Desenvolvimento Humano (IDH) 0,956 de Copacabana, o bairro que ainda concentra o maior número de casos e óbitos, para outras com IDH abaixo de 0,750, como muitos bairros da AP3 (Maré, Complexo do Alemão, Jacaré, Parada de Lucas, Manguinhos, Acari, etc.) e da AP5 (como Santa Cruz), assim como para os municípios da Baixada, onde o IDH vai de 0,753 (Nilópolis) a 0,659 (Japeri). Como é possível observar, a expansão, o impacto e as estratégias de contenção do coronavírus não é democrática, nem socialmente, nem economicamente, nem tampouco espacialmente.

\section{CONSIDERAÇÕES FINAIS}

Com o avanço da Pandemia, urge a necessidade de pensar os elementos que envolvem a produção geográfica das sociedades, bem como a e interlocução dos processos e condição dos espaços para analisarmos os efeitos da COVID-19. A compreensão da complexidade da periferia, em sua forma-conteúdo, constitui-se, portanto, um desafio para o planejamento urbano e para construção de políticas públicas equânimes.

Entender os caminhos da COVID-19 na Baixada Fluminense, como uma periferia urbana, serve para vislumbrarmos os efeitos sobre as populações mais pobres bem como entender processos espaciais como a seletividade, a fragmentação dos espaços, e a segregação. Uma vez que os dados apontados nestes primeiros meses [março, abril e maio] de avanço da doença revelam uma geografia própria da doença que não se restringe a uma elite econômica e viajada. Assim, quebra-se o mito de que a COVID-19 é uma doença democrática. Ao contrário, ela tende a expandir-se para áreas mais pobres, sem infraestrutura de saúde e causando graves transtornos sociais nestas localidades.

Por isso, tornou-se exigência de pesquisadores que estão analisando a realidade da pandemia no Brasil combater a relativização do número anunciado de óbitos pelas estruturas governamentais e a supremacia das preocupações econômicas sobre as próprias estratégias de contenção do vírus. Tais ações governamentais reforçam o desprezo pela vida - especialmente dos mais pobres, negros e segregados territorialmente - de certas autoridades. Assim como mundialmente a COVID-19 tem sido frequentemente atribuída às práticas culturais "impuras" dos chineses pobres de Wuhan (COLETIVO CHUANG, 2020), em tempos de individualismo, concorrência mercadológica, preconceitos exacerbados e intolerância política, há a sincera preocupação que se culpabilize, na Baixada Fluminense, as próprias vítimas.

Assim, compreender os caminhos do coronavírus na Baixada Fluminense exige clareza e profunda organização das informações desde a coleta dos dados. Por sua vez, observar as tendências e eixos espaciais permite que as prefeituras e o governo estadual possam orientar suas políticas para o interior dos municípios - os casos da COVID-19 já avançam para bairros e sub-bairros mais afastados - e sejam capazes de enfrentar 
os graves problemas destes espaços, pois muitas destas localidades não possuem equipamentos médicos e nem a assistência necessária ao tratamento da doença. Reforçamos, acima de tudo, que são lugares que certamente necessitam de muito apoio do poder público diante da vulnerabilidade estrutural a qual estão expostas.

\section{REFERÊNCIAS}

ABREU, Maurício. Evolução Urbana do Rio de Janeiro. 4ạ edição. IPP,2013, 156p.

AGNEW, J. American "Populism" and the Spatial Contradictions of US Government in the Time of Covid-19. Geopolítica(s). Revista de Estudios Sobre Espacio y Poder, 11(Especial), 15-23, 2020. Disponível em < https://doi.org/10.5209/geop.69018>. Acesso em 10 de maio de 2020.

BARBOSA, Jorge Luiz. Por uma quarentena de direitos para as favelas e as periferias!. Espaço e Economia [Online], 17 | 2020. Disponível em < https://doi.org/10.4000/espacoeconomia.10274 > . Acesso em 15 de abril de 2020.

CASTILHO, D. Um vírus com DNA da globalização: o espectro da perversidade. Espaço e Economia [Online], 17 | 2020, Disponível em < https://doi.org/10.4000/espacoeconomia.10332 > Acesso em 19 maio 2020.

COLETIVO CHUANG. Contágio Social: coronavírus e a luta de classes microbiológica na China. São Paulo: Veneta, 2020.

CORREA, Roberto Lobato. Trajetórias Geográficas. Rio de Janeiro. Bertrad Brasil, 2005, 304p.

DAVIS, Mike, et al. Coronavírus e a luta de classes. Terra sem Amos: Brasil, 2020.Disponível em: <

https://terrasemamos.files.wordpress.com/2020/03/coronavc3adrus-e-a-luta-de-classes-tsa.pdf.> Acesso 10 de abril de 2020.

FARIAS, Heitor Soares de. O avanço da Covid-19 e o isolamento social como estratégia para redução da vulnerabilidade. Espaço e Economia, 17 | 2020. Disponível em < https://doi.org/10.4000/espacoeconomia.11357>. Acesso em 15 maio 2020.

GEIGER, Pedro; SANTOS, Ruth Lyra. Notas sobre a evolução da ocupação humana da Baixada Fluminense. Revista Brasileira de Geografia - IBGE. Rio de Janeiro, n. 3 - Ano XVI, Jul/set, 1955.

HAESBAERT, Rogério: Entre a contenção e o confinamento dos corpos-território: reflexões geográficas em tempos de pandemia (I) e (II). AGB-Campinas, 24.03.2020. Disponível em: http://agbcampinas.com.br/site/2020/rogerio-haesbaert-desterritorializacao-semlimites-reflexoes-geograficas-em-tempos-de-pandemia-i/

MONIÉ, Frédéric. A África subsaariana diante da pandemia de Coronavírus/Covid-19: difusão espacial, impactos e desafios. Espaço e Economia [Online], 18 | 2020. Disponível em < https://doi.org/10.4000/espacoeconomia.13629 >. Acesso em 22 abril 2020.

MONTEMERLI, Roberto. Os desafios da Itália na emergência do coronavírus . Espaço e Economia [Online], 17 | 2020. Disponível em < http://journals.openedition.org/espacoeconomia/11312>. Acesso em 08 abril 2020.

LAGO, Luciana Corrêa. A "periferia" metropolitana como lugar do trabalho: da cidade - dormitório à cidade plena. In: Cadernos IPPUR /UFRJ /Instituto de Pesquisa Planejamento Urbano e Regional da Universidade Federal do Rio de Janeiro. Ano XXI, no 2, p. 9 28, ago-dez, 2007

LOYER, B; GIBLIN, B. (2020). ¿Qué mundo geopolítico después de 2020?. Geopolítica(s). Revista De Estudios Sobre Espacio Y Poder, 11(Especial), 115-126. Disponível em < https://doi.org/10.5209/geop.69250 >. Acesso em 15 de maio de 2020.

RÍOS SIERRA, J. La inexistente respuesta regional a la Covid-19 en América Latina. Geopolítica(s). Revista de Estudios Sobre Espacio Y Poder, 11(Especial), 209-222, 2020

OLIVEIRA, Leandro Dias. A emersão da região logístico - industrial do Extremo Oeste Metropolitano fluminense: reflexões sobre o processo contemporâneo de reestruturação territorial - produtiva, Espaço e Economia, 7, 2015. Disponível em< http://journals.openedition.org/espacoeconomia/1814>. Acesso em 15 de maio de 2020.

ROCHA, André Santos da. Os efeitos da reestruturação econômica metropolitana na Baixada Fluminense: Apontamentos sobre o "novo" mercado imobiliário da região. Espaço e Economia. Ano 3, n. 6, Janeiro / Junho de 2015. Disponível em: http://espacoeconomia.revues.org/1677. Acesso em: 10 de dezembro de 2015. 
Reestruturação Urbana e o “Novo Mercado Imobiliário” Em Nova Iguaçu (RJ): Revista Continentes, n. 14, p. 629, ago. 2019. Disponível em: <http://www.revistacontinentes.com.br/continentes/index.php/continentes/article/view/232>. Acesso em 29 maio 2020.

. Globalização, gestão e acesso aos sistemas público e privado de saúde: a Baixada Fluminense no contexto da pandemia. Espaço e Economia.18 | 2020. Disponível em < https://doi.org/10.4000/espacoeconomia.12672 >. Acesso em 20 de abril de 2020.

SANTOS, Milton. 0 trabalho do Geógrafo no Terceiro Mundo. 5o ed. São Paulo, 2009. p.133.

SANTOS, Simone M., SOUZA, Wayner V. (org.) Introdução à estatística espacial para a saúde pública. Série B. Textos Básicos de Saúde / Série Capacitação e Atualização em Geoprocessamento em Saúde, vol. 3 Brasília: Ministério da Saúde, 2007. Disponível em: http://www.escoladesaude.pr.gov.br/arquivos/File/TEXTOS_CURSO_VIGILANCIA/capacitacao_e_atualizacao_em_geoprocessamento _em_saude_3.pdf.> Acesso em 15 de abril de 2020.

SEGADA SOARES, Maria Teresinha. "Nova Iguaçu: absorção de uma célula urbana pelo Grande Rio de Janeiro. In: Revista Brasileira de Geografia. Rio de Janeiro: IBGE, vol2, n24, 1962.

SIMÕES, Manoel Ricardo. A cidade Estilhaçada - reestruturação econômica e emancipações municipais na Baixada Fluminense. Mesquita: Entorno, 2007.

SOUZA, Marcelo Lopes. ABC do desenvolvimento Urbano. 3a Ed. Rio de janeiro: Bertrand Brasil, 2007. 192p.

WACQUANT, Loïc D. Los condenados de la ciudad: Gueto, periferias y Estado. Siglo Veintiuno, 2007.

WACQUANT, Loïc D. Punishing the Poor. The Neoliberal Government of Social Insecurity. Durham, 2009.

ZAAR, Miriam Hermi; GARCÍA ÁVILA, Manuel-Blas. El Covid-19 en España y sus primeras consecuencias. Espaço e Economia. [Online], 17 | 2020. Disnponíevel em < https://doi.org/10.4000/espacoeconomia.10142>. Acesso em 19 maio 2020. 